\title{
Boundary bound states in the Bose-Hubbard-like chain
}

\author{
A.A. Zvyagin \\ B. Verkin Institute for Low Temperature Physics and Engineering of the National Academy of Sciences of Ukraine \\ 47 Lenin Ave., Kharkov 61103, Ukraine \\ E-mail: zvyagin@ilt.kharkov.ua
}

Received August 19, 2006

\begin{abstract}
The degenerate Hubbard-like chain with open boundary conditions is studied with the help of the Bethe ansatz. The special case of the Bose-Hubbard-like chain is studied in detail. Boundary bound states, which appear as the consequence of the local potential(s), applied to the edge(s) of the open chain are studied in the ground state.
\end{abstract}

PACS: 05.30.Jp Boson systems (for static and dynamic properties of Bose-Einstein condensates); 71.10.-w Theories and models of many-electron systems.

Keywords: Bose-Hubbard chain, boundary bound states.

Last decade the interest in the behavior of low-dimensional interacting quantum systems has been grown considerably. In particular, the interest in the studies of interacting lattice bosonic systems is connected with the experimental realization of such a systems, e.g., in systems of ultracold gases of atoms in optical lattices, see, e.g., $[1,2]$, in some of which the Bose-Einstein condensation is believed to be observed. Here we consider a Bose-Hubbard-like model, which properties can be obtained using non-perturbative methods.

Several authors studied the properties of the so-called degenerate Hubbard-like chain with the help of the Bethe ansatz, see, e.g., [3-11]. The Hamiltonian of the model was proposed first in the form

$\mathcal{H}_{p}=\sum_{j=1}^{L}\left[\sum_{s=1}^{N} \mathscr{P}\left(c_{j, s}^{\dagger} c_{j+1, s}+\right.\right.$ H.c. $\left.) \mathscr{P}+U \sum_{s, s^{\prime}=1\left(s \neq s^{\prime}\right)}^{N} n_{j, s} n_{j, s^{\prime}}\right]$,

where $c_{j, s}^{\dagger}\left(c_{j, s}\right)$ creates (destroys) a particle at site $j$ with the spin index $s, n_{j, s}=c_{j, s}^{\dagger} c_{j, s}, U \geq 0$ is the Hubbard repulsion constant, and the hopping integral is taken to be equal to unity. In particular, for $N=2$ one deals with the standard Hubbard chain of interacting electrons. On the other hand, the case $N \rightarrow \infty$ has to be related to the so-called Bose-Hubbard model: each particle has infinite number of states. The operator $\mathcal{P}$ projects onto the subspace of states having at most two particles at each site.
The presence of such a projector is crucial for the applicability of the Bethe ansatz method (first studies of the model, e.g., $[3,4]$ missed that fact). Bethe ansatz gives the possibility to find eigenvalues and eigenfunctions of the stationary Schrödinger equation of the considered model. Eigenvalues and eigenfunctions are parametrized by, generally speaking, several sets of quantum numbers (one set for each degree of freedom), called rapidities [12].

Later, however, it was recognized that it was not enough to use the projectors. For example, for $U=0$ Bethe ansatz describes free particles, while according to Eq. (1) there must be correlations between particles, caused by the projectors. It was shown [8] that the continuum limit of the scattering phase shifts, which follow from the Bethe ansatz equations, are those of particles, interacting via a potential of the form $1 / \sinh ^{2} r$ (where $r$ is the distance between particles). It implies the necessity to include a long-range interaction, which dynamically exclude many-particle configurations at each site, to the Hamiltonian Eq. (1). However, unfortunately, the precise form of the lattice Hamiltonian of the degenerate Hubbard-like model, which can be solved by the Bethe ansatz, has not been found yet. Nevertheless, studies of the Bethe ansatz solvable model are very important (even in the absence of the precise form of the lattice Hamiltonian), because the features of the Bethe ansatz exact solution, e.g., for the Bose-Hubbard-like case $N \rightarrow \infty$ are reminiscent of those for the real lattice Bose-Hubbard model, which was con- 
sidered, e.g., in the mean-field approximation [13], or by the quantum Monte-Carlo techniques [14]. The most interesting feature of the lattice Bose-Hubbard-like chain (and, in fact, of the degenerate Hubbard-like chain for $N>2$ ) is the quantum phase transition at the critical value of the couling constant $U_{c}$ between a metallic (superfluid) gapless phase and an insulator gapped one. This feature is absent for the standard electron Hubbard chain $N=2$ (where $U_{c}=0$ ). However, the Bethe ansatz solution of the degenerate Hubbard-like chain [3-10] reveals such a feature.

Most of the previous Bethe ansatz studies of the model were related to the chain with periodic boundary condi- tions (i.e. $L+1 \rightarrow 1$ etc.). Here we consider the chain with open boundary conditions, i.e. one has to omit the term with $\left(c_{L, s}^{\dagger} c_{1, s}+\right.$ H.c. $)$ from the Hamiltonian (1). Also, we add the boundary potential $-p \sum_{s=1}^{N} n_{1, s}$ to the considered Hamiltonian (in fact one can add another boundary potential, which acts on the particles at the last site, but for simplicity we limit ourselves with only one boundary potential) without destroying of the exact integrability. Bethe ansatz equations, for several sets of rapidities, which parametrize eigenfunctions and eigenvalues of the considered system, have the form

$$
\begin{gathered}
\exp \left(2 i k_{j} L\right) B\left(k_{j}\right)=\prod_{\beta=1}^{M_{1}} e_{1}\left(\sin k_{j}-\lambda_{\beta}^{(1)}\right) e_{1}\left(\sin k_{j}+\lambda_{\beta}^{(1)}\right), j=1, \ldots, N_{p}, \\
\prod_{\beta=1}^{M_{s-1}} e_{1}\left(\lambda_{\alpha}^{(s)}-\lambda_{\beta}^{(s-1)}\right) e_{1}\left(\lambda_{\alpha}^{(s)}+\lambda_{\beta}^{(s-1)}\right) \prod_{\beta=1}^{M_{s+1}} e_{1}\left(\lambda_{\alpha}^{(s)}-\lambda_{\beta}^{(s+1)}\right) e_{1}\left(\lambda_{\alpha}^{(s)}+\lambda_{\beta}^{(s+1)}\right)= \\
=\prod_{\beta=1}^{M_{s}} e_{2}\left(\lambda_{\alpha}^{(s)}-\lambda_{\beta}^{(s)}\right) e_{2}\left(\lambda_{\alpha}^{(s)}+\lambda_{\beta}^{(s)}\right), \quad \alpha=1, \ldots, M_{s}, \quad s=1, \ldots, N-1,
\end{gathered}
$$

where $B\left(k_{j}\right)=\left[\exp \left(i k_{j}\right)-p\right] /\left[\exp \left(-i k_{j}\right)-p\right], \quad e_{n}(x)=$ $=(2 x+i U n) /(2 x-i U n), N_{p}$ is the number of particles, $M_{0} \equiv N_{p}$, and $\lambda_{j}^{(0)} \equiv \sin k_{j}$. The energy of the corresponding state is equal to

$$
E=-2 \sum_{j=1}^{N_{p}} \cos k_{j}-p N_{1},
$$

where $N_{1}$ is the total number of particles at the first site of the chain. Obviously, the Bethe ansatz equations (2), (3) coincide with the known ones for the case of free particles with $N$ internal degrees of freedom $(U=0$, from which case one can see that Bethe ansatz equations are quantization conditions), for the case of free spinless fermions (orthofermions) $U \rightarrow \infty$, and with the ones for the standard Hubbard chain for $N=2$ [12]. In the continuum limit of small $k_{j}$ (here we replace $\cos k_{j} \rightarrow$ $\rightarrow 1-k_{j}^{2} / 2$ and $\sin k_{j} \rightarrow k_{j}$ the Bethe ansatz equations agree with the ones for particles with $\delta$-function local interactions between particles [15].

Below we shall consider only the case $N \rightarrow \infty$, related to the Bose-Hubbard chain with open boundary conditions. In this case only one set of quantum numbers, $k_{j}$ is essential [4]. In the thermodynamic limit $L \rightarrow \infty, N_{p} \rightarrow \infty$, but with the ratio $N_{p} / L$ being fixed, using the standard for Bethe ansatz solvable models procedure [12], we can write down integral equations for so-called «dressed ener- gies» $\varepsilon(k)$ and densities $\rho(k)\left(\rho_{h}(k)\right.$ is the density of holes $)$ for eigenstates

$$
\begin{gathered}
\varepsilon(k)+2 \cos k+\mu=\int_{-Q}^{Q} d k^{\prime} \cos k^{\prime} G\left(\sin k-\sin k^{\prime}\right) \varepsilon(k), \\
\rho(k)+\rho_{h}(k)=\frac{1}{2 \pi}+\frac{1}{2 L}\left[-2 \cos k G(2 \sin k)+i \frac{B^{\prime}(k)}{2 \pi \cos k B(k)}\right]+ \\
+\cos k \int_{-Q}^{Q} d k^{\prime} G\left(\sin k-\sin k^{\prime}\right) \rho(k),
\end{gathered}
$$

where $\mu$ is the chemical potential, the kernel has the form

$$
G(x)=\frac{2 U}{\pi\left[4 x^{2}+U^{2}\right]} .
$$

Here, using the fact that dressed energies, densities, kernels and driving terms (those, which do not depend on $\varepsilon(k), \rho(k)$, and $\rho_{h}(k)$ are even functions, we replaced distributions of $k$ over total intervals, like for periodic chains, instead of half-intervals for the open chain. This is why, the term $G(2 \sin k) / L$ appeared in the equation for densities. One can see that driving terms in the equations for densities has the term of order of 1 and the contribution of order of $1 / L$. The integral equation for densities is linear. Hence, we can look for the solution of that equation in the form $\rho(k)=\rho^{(0)}(k)+(1 / L) \rho^{(1)}(k)$. Equations 
for $\rho^{(0)}$ and $\rho^{(1)}$ are separated from each other, and they describe the behavior of the bulk and boundaries, respectively.

Obviously, the equations for the bulk part, $\rho^{(0)}$, coincide with the ones for periodic case [3-11]. Quantum numbers $k$ are distributed in the interval $-Q \leq k \leq Q$. It is easy to see that for $U<U_{c}=4 \sqrt{3}$ the considered model is in the metallic phase with gapless low-lying excitations, while for $U>U_{c}$ the model is in the insulating phase $[4,8]$. From now on we concentrate in the insulating case with $Q=\pi\left(N_{p}=L\right)$. In that case it is easy to calculate the ground state energy (of the bulk), which is equal to

$$
E_{G}=-4 \int_{0}^{\infty} \frac{d \omega J_{0}(\omega) J_{1}(\omega)}{\omega} \mathrm{e}^{-U \omega / 2}=-\frac{8}{\pi f} \mathbf{E}(f)+U,
$$

where $f=4 /\left[\sqrt{U^{2}+16}\right]$, and $\mathbf{E}$ is the complete elliptic integral of the second kind. The energy of the low-lying excitation is

$$
E_{h}=2 \cos k_{0}+\mu+4 \int_{0}^{\infty} \frac{d \omega J_{1}(\omega)}{\omega} \cos \left(\omega \sin k_{0}\right) \mathrm{e}^{-U \omega / 2},
$$

where $k_{0}$ is related to the momentum of the excitation $p_{h}$ as

$$
p_{h}=2 \int_{0}^{k_{0}} d k\left[\frac{1}{2}+\cos k \int_{0}^{\infty} d \omega \cos (\omega \sin k) J_{0}(\omega) \mathrm{e}^{-U \omega / 2}\right] .
$$

It is easy to see that $E_{h}$ is the monotonically decreasing function of $\left|k_{0}\right|$, and it has minima at $k_{0}= \pm \pi$ and a maximum at $k_{0}=0$. Hence, the low boundary of the band of low-lying excitation is equal to $-2+8 /\left[U+\sqrt{U^{2}+4}\right]$, and the upper boundary is equal to $2+8 /\left[U+\sqrt{U^{2}+4}\right]$. As for the chemical potential, it is then equal to $\mu=-E_{h}\left(k_{0}=\pi\right)$. Other bulk excitations (bound states, or strings) have higher energies.

Now let us consider the boundary contribution. In the absence of the boundary potential the ground state energy of free edges, or the surface energy (it is the difference between the ground state energy of the chain with open and with periodic boundary conditions), is equal to

$$
\begin{gathered}
E_{s}=-2 U+2 U \sqrt{U^{2}+4}-\frac{i}{2 \pi} \int_{-\pi}^{\pi} d k \cos ^{2} k \times \\
\times\left[\sqrt{(\sin k+i(U / 4))^{2}-1}-\sqrt{(\sin k-i(U / 4))^{2}-1}\right] .
\end{gathered}
$$

Next, we turn to boundary bound states. In principle, one is free to leave boundary bound states empty [12]. However, they are important for, e.g., calculation of the exponents for correlation functions. There are no boundary bound states for $|p|<1$, and we can use the previous analysis in that case. On the other hand, for $|p|>1$ boundary bound states, caused by the boundary potential $p$ appear with the roots $k_{j}= \pm i \ln p$ and the energy $E_{b}=-\left(p^{2}+1\right) / p$. The (most important) levels of the low-energy boundary bound states are split off the upper and lower boundaries of the band of the low-lying excitations Eq. (7). Higher energy boundary bound states are also split off upper and lower boundaries of the bands for higher energy bulk excitations. Notice that we are interested in decaying roots. The surface energy in the case with $p \neq 0$ is equal to

$$
\begin{gathered}
E_{s b}=E_{s}-p N_{1}+E_{b} \theta(|p|-1)+\int_{-\pi}^{\pi} d k \cos ^{2} k \times \\
\times\left[\frac{i B^{\prime}(k)}{2 \pi \cos k B(k)}-G(\sin k-i \ln p)-G(\sin k+i \ln p)\right] .
\end{gathered}
$$

The last two terms denote the removing of the root, related to the boundary bound state, from the continuum of bulk states, forming the vacuum.

On the other hand, Eqs. (2) can be solved for the cases of free bosons $(N \rightarrow \infty, U=0)$, or free spinless fermions (orthofermions) $(U \rightarrow \infty)$ [12]. Those cases are interesting, because we can understand the behavior of the boundary bound states for the metallic case with gapless excitations. Bethe ansatz equations for these two cases have the form

$$
\exp \left(2 i k_{j}^{L}\right) B\left(k_{j}\right)= \pm 1
$$

where the «plus» sign is taken for the first case, and the «minus» one for the second case, respectively. Here one can obviously see that Bethe ansatz equations are quantization conditions for the rapidities $k_{j}$ (they are quasi-momenta for free particles). One can also see that for $p \neq 0(p>1)$ solutions for boundary bound states appear even for free particles (they are split off the bands of bulk linear excitations). The surface energy at $N_{p}=L$ is equal to

$$
E_{s p}^{(f)}=-p N_{1}+E_{b} \theta(|p|-1)-\frac{1}{2 \pi} \int_{0}^{\infty} d \omega \frac{\sinh [2-2 S(p)]}{\sinh (2 x) \cosh (x)},
$$

where $S(p)=(2 / \pi) \arctan [(1+p) /|1-p|]$. The surface energy for $p=0$ is equal to $E_{S}=1-(2 / \pi)$. It turns out that it is incorrect to take the limit $U$ of Eqs. (9) and (10) to obtain this result, because those equations were obtained for the insulating phase $U>U_{c}$.

In summary, we have studied the Bethe ansatz solution of the degenerate Hubbard-like chain with open boundary 
conditions. The most interesting case of the Bose-Hubbard-like chain is analysed for the ground state. The surface energy (the energy of the free edges of the chain) is calculated. We have shown that for large values of boundary potentials boundary bound states are split off the bands of the bulk states. The most important states appear in the gap, where no bulk excitations exist for the insulating phase with $U>U_{c}$. Our results can be applied, e.g., for ultracold atoms in optical lattices, or photonic lattices, where discrete surface low-lying excitations were recently observed [16-19].

1. T. Stöferle, H. Moritz, C. Schori, M. Köhl, and T. Esslinger, Phys. Rev. Lett. 92, 130403 (2004).

2. B. Paredes, A. Widera, V. Murg, O. Mandel, S. Fölling, I. Cirac, G.V. Shlyapnikov, T.W. Hänsch, and I. Bloch, Nature 429, 277 (2004).

3. T.C. Choi, Phys. Lett. 80A, 49 (1980).

4. F.D.M. Haldane, Phys. Lett. 80A, 281 (1980).

5. F.D.M. Haldane, Phys. Lett. 81A, 545 (1981).

6. T.C. Choi and F.D.M. Haldane, Phys. Lett. 90A, 83 (1982).

7. K. Lee and P. Schlottmann, Phys. Rev. Lett. 63, 2299 (1989).
8. P. Schlottmann, Phys. Rev. B43, 3101 (1991).

9. A.A. Zvyagin, Fiz. Nizk. Temp. 17, 1436 (1991) [Low Temp. Phys. 17, 779 (1991)].

10. P. Schlottmann, Phys. Rev. B45, 5784 (1992).

11. H. Frahm and A. Schadschneider, J. Phys. A: Math. Gen. 26, 1463 (1993).

12. See, e.g., A.A. Zvyagin, Finite Size Effects in Correlated Electron Systems: Exact Results, Imperial College Press, London (2005).

13. G. Kotliar and A. Ruckenstein, Phys. Rev. Lett. 57, 1362 (1986).

14. G.G. Batrouni, R.T. Scalettar, and G.G.T. Zimanyi, Phys. Rev. Lett. 65, 1765 (1990).

15. P. Schlottmann, Int. J. Mod. Phys. B11, 355 (1997).

16. Ya.V. Kartashov, V.V. Vyslouh, and L. Torner, Phys. Rev. Lett. 96073901 (2006).

17. C.R. Rosberg, D.N. Neshev, W. Krolikowski, A. Mitchell, R.A. Vicencio, M.I. Molina, and Yu.S. Kivshar, unpublished.

18. M. Matuszewski, W. Kvolikowski, M. Trippenbach, and Yu.S. Kivshar, Phys. Rev. A73, 063621 (2006).

19. B. Eirmann, Th. Anker, M. Albiez, M. Taglieber, P. Treutlein, K.-P. Marzlin, and M.K. Oberthaler, Phys. Rev. Lett. 92, 230401 (2004). 